\title{
Discontinuous density gradient sperm processing concentrates normal morphological spermatozoa better in the yield: An observational study
}

\author{
Vijayakumar C N ${ }^{1, *}$, Sandeep Tripathi², Surabhi Tomar Sharma ${ }^{3}$ \\ ${ }^{1}$ Ph. D. Scholar, ${ }^{2,3}$ Assistant Professor, Dept. of Obstetrics and Gynecology, School \& Advanced Life Sciences, NIMS \\ University, Jaipur, Rajasthan, India
}

*Corresponding Author:

Email: vizchelur@yahoo.com

\begin{abstract}
Aim: To evaluate the efficiency of Discontinuous Double Density Gradient Sperm Preparation over Swim up preparation in concentrating normomorphic spermatozoa in the yield.

Materials and Methods: 50 semen samples obtained from patients visiting the NIMS Fertility and Research Center were analyzed for normal morphology prior to processing, then the sample was aliquoted in to two equal halves, each of it was processed by Swim up and Discontinuous Density Gradient preparation simultaneously. Yields obtained from the both methods were tested for normal morphology of sperms using Diff quick staining method and micrometry according to WHO Manual 2010.

Results: Analyzed neat semen samples showed mean Sperm concentration of $38.2 \mathrm{~m} / \mathrm{ml}$ and mean sperm morphology of $7.54 \%$. Post processing yield from Swim up preparation had mean concentration of $30.08 \mathrm{~m} / \mathrm{ml}$ and normal morphology of $8.66 \%$ (pvalue 0.05 ) where as the yield from discontinuous density Gradient preparation showed up a mean concentration of $32.2 \mathrm{~m} / \mathrm{ml}$ and normal morphology of $9.8 \%$ (p-value 0.001 )

Conclusion: From the study it was evident that the Discontinuous Density Gradient Sperm Preparation method has a clear edge over Swim up preparation in concentrating normal morphological spermatozoa in the yield
\end{abstract}

Keywords: Sperm Processing, Normal morphology, Swim up technique, Density gradient technique.

\section{Introduction}

Semen Analysis is the only gold standard assessment for the male factor in the course of evaluation of the infertile couple. There is a strong negative correlation of analyzed semen parameters with published reference values of WHO 2010., ${ }^{1,2}$ Off the numerous parameters that can be tested in a given sample four parameter are more important and correlate independently, negatively to fecundity, ${ }^{3}$ they are,

1. semen volume

2. concentration of spermatozoa with in the semen

3. their motility and grade

4. their morphology

Since, the spermatozoa with in a sample are highly pleomorphic in nature, its standardized analysis is always called for. ${ }^{4}$ WHO from it third edition in 1992 to till date has changed the acceptable normal thresholds very widely like in 1999 4th edition the normal morphology was about $30 \%$ normal forms in the given sample to accept analyzed sample as normal, but, now in 5th edition (2010) it has brought it down to $\geq$ $4 \%$ as normal. ${ }^{5,6}$ WHO has moved away from microscopic morphological examination in 1999 to morphometric examination to bring about objectivity to the semen morphology examination. ${ }^{2}$

Abnormal semen morphology has been found to be associated with poor pregnancy rates, poor implantation rates, poor embryo grading, increased miscarriage, reduced live birth rate. ${ }^{7-10}$ Hence, more and more focus of treating physicians' are turning towards better sperm selection techniques to enhance the chance of morphologically normal sperms being used in the treatment of infertile couple, thus, increasing the chances of suggesting Intra Cytoplasmic Sperm Injection (ICSI) more over to other methods of assisted conception to the aspiring infertile couple, whose spermiogram shows high percentages of abnormal sperms.

Spermatozoa that are normal and motile must be separated from seminal plasma prior to use in the process of fertilization. Separating the spermatozoa from seminal plasma induces in them the process of capacitation and capacitated sperms can only fertilize the ova. In vivo the separation of motile spermatozoa results as a concerted effort of cervical mucus and sperms own motility. In vitro the separation of motile and normal spermatozoa is to be performed by trained personnel, which are referred to as sperm preparation process.

There are various direct and indirect methods of processing the semen sample for assisted reproductive technology purposes, largely the methods utilize the sperm's motility as the selection criterion. ${ }^{2,11}$ Depending upon the movement of sperms they are classified as

1. Swim Up $\mathrm{p}^{12}$ and

2. Swim Down Techniques. ${ }^{13}$

In Swim Up the motile sperms migrate in to the media phase layered above the sample phase against gravitational force, hence, named as Swim Up. 
In Swim Down the sperms move down towards the bottom of the column of media along the gravitational force, with or without assistance from centrifugation force (denoted as ' $\mathrm{g}$ ' force). To desist the movement of sperms, obstacles in the form of inert colloidal particles/ Glass wool/ porous substances are utilized in the column, thus, filtering off the non motile population from the final yield

There are also special techniques like Magnetic Assorted Cell Sorting, Flow Cytometry etc., that can be used to separate normal spermatozoa from the mixed population but, these methods are very expensive in terms of instrumentation and running cost. ${ }^{14-16}$ Hence, widely used methods in most of the laboratories/ hospitals around the world for sperm processing are namely

1. Wash and Swim Up (WSC) (popular as Swim Up technique) and

2. Discontinuous Double Density Gradient (DGC) (popular as Density Gradient technique) which are simple yet effective in getting concentrated motile sperms in the yield.

Not all the motile sperms will be normal morphologically. ${ }^{17,18}$ But, practitioners swear by the logical statements rather than the experimental evidences as to the processing method's capabilities to concentrate normal morphological sperms in the final yield.

Hence, we designed this small observational study to demonstrate the usefulness of discontinuous double density gradient technique's capabilities to concentrate better morphological sperms in comparison to Swim up technique with in the same population.

\section{Materials and Methods}

50 semen samples from the male patients visiting to the NIMS Fertility \& Research Center for IUI/ IVF were used for this study. Informed consents were obtained from the participating patients.

The neat semen samples were first analyzed for concentration, Motility using Makler's counting chamber (Safi Medical Instruments, Israel) and Morphometric evaluation under 1000x oil immersion after the smears were stained using diff quick stain (Sperm Processor, Aurangabad, India).

Then the samples were divided in to two equal halves and each aliquot was processed simultaneously and independently. One aliquot was processed using Swim Up method (using Vitromed ${ }^{\mathrm{TM}}$ HEPES Medium) that of the other aliquot using Discontinuous Double Density Gradient method using ready to use Gradients $80 \%$, Gradient $40 \%$ and Vitromed TM HEPES medium.

Swim Up Method (WSC): Semen Sample was mixed with 1:3 volumes of equilibrated HEPES medium in a sterile round bottom tube and centrifuged at $250 \mathrm{~g}$ for 10 min, resulting pellet was carefully layered with $2 \mathrm{ml}$ equilibrated HEPES medium, incubated at an angle for $60 \mathrm{~min}$, the turbid supernatant was aspirated in to a new sterile round bottom tube and was centrifuged at $250 \mathrm{~g}$ for $10 \mathrm{~min}$ resultant pellet was resuspended in $0.5 \mathrm{ml}$ equilibrated HEPES medium. ${ }^{19}$

Discontinuous Density Gradient Method (DGC): A discontinuous density gradient column of $40 \%$ over $80 \%$ in a sterile conical tube was prepared by under laying $1 \mathrm{ml}$ of $80 \%$ gradient below $1 \mathrm{ml}$ of $40 \%$ Gradient. Semen sample was gently layered over the $40 \%$ gradient, the column was centrifuged at $300 \mathrm{~g}$ for $15 \mathrm{~min}$, carefully the pellet was aspirated in to a round bottom tube containing $3 \mathrm{ml}$ of equilibrated HEPES medium, mixed well and centrifuged at $250 \mathrm{~g} 10 \mathrm{~min}$, resultant pellet was resuspended in $0.5 \mathrm{ml}$ of fresh equilibrated HEPES medium. ${ }^{20}$

The resultant yields were analyzed for motility concentration using Makler's Counting Chamber and morphometric evaluations on dried smears stained with DiffQuick Staining. The results were analyzed using Graph pad In Stat Trial version

\section{Results}

The neat semen samples showed a mean concentration of 38.2 millions/ $\mathrm{ml}$ (SD of 22.12 and SEM of 3.12). Yield analysis of Wash and Swim Up group showed concentration of 30.08 millions/ ml (SD 18.89 and SEM 2.69) where as the Density Gradient Group showed the concentration of 32.2 millions/ ml (SD 19.73 and SEM 2.79).

The neat semen samples showed a mean \% normal morphology of $7.54 \%$ against WSC Group with $8.66 \%$ (p-Value $\left.0.05^{* *}\right)$ and with that of DGC Group with $9.8 \%$ (p-Value $0.001 * * *)$.

Table 1

\begin{tabular}{|c|l|l|l|l|}
\hline Parameters & \multicolumn{1}{|c|}{$\begin{array}{c}\text { Neat } \\
\text { Semen }\end{array}$} & $\begin{array}{c}\text { Wash+Swim } \\
\text { Up } \\
\text { Group }\end{array}$ & $\begin{array}{c}\text { Density } \\
\text { Gradient } \\
\text { Group }\end{array}$ & Significance \\
\hline No of Samples & 50 & 50 & 50 & $\begin{array}{l}\text { Autologous } \\
\text { Sibling Sample }\end{array}$ \\
\hline
\end{tabular}




\begin{tabular}{|l|l|l|l|l|}
\hline $\begin{array}{l}\text { Mean } \\
\text { in millions/ ml }\end{array}$ & $\begin{array}{l}38.2 \\
\text { SD 22.12 } \\
\text { SEM 3.12 }\end{array}$ & $\begin{array}{l}30.08 \\
\text { SD 18.89 } \\
\text { SEM 2.69 } \\
(\mathrm{p}=0.05 \mathrm{~ns})\end{array}$ & $\begin{array}{l}\text { S2.2 } \\
\text { SD 19.73 } \\
\text { SEM 2.79 } \\
(\mathrm{p}=0.15 \mathrm{~ns})\end{array}$ & Not significant \\
\hline $\begin{array}{l}\text { Mean } \\
\text { Morphology } \\
\text { in \% }\end{array}$ & $7.54 \%$ & $\begin{array}{l}8.66 \% \\
(\mathrm{p}=0.05 \mathrm{~ns})\end{array}$ & $\begin{array}{l}9.8 \% \\
(\mathrm{p}=0.001 * * *)\end{array}$ & Significant \\
\hline
\end{tabular}

Graph 1: Comparison of Mean Semen Count

\section{Comparision of Mean Semen Count}

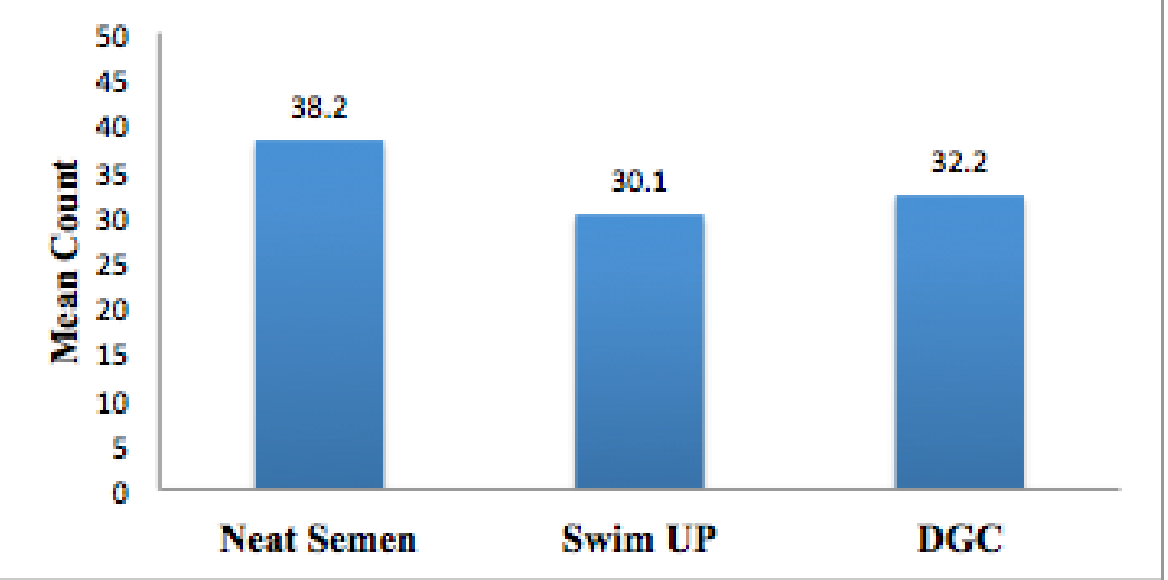

Graph 2: Comparison of Normal Morphology

\section{Comparision of Normal Morphology}

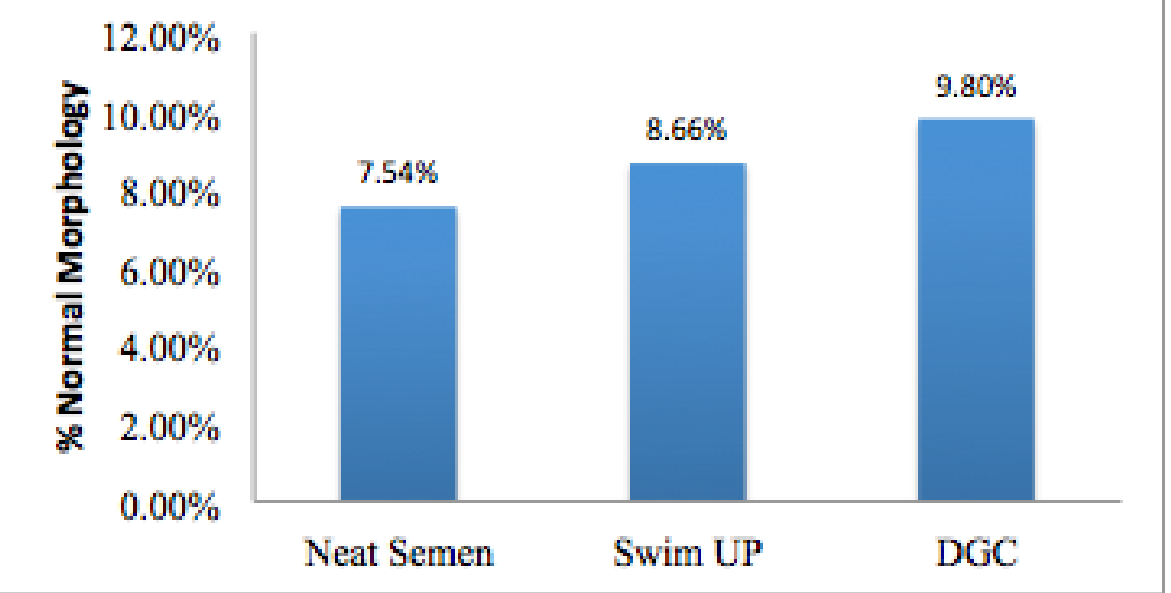

\section{Discussion}

From the results it was evident that both methods of sperm preparation are effective in concentrating the motile spermatozoa in the yield. Motile spermatozoa in yield are very useful in the treatment of infertile couple by ART including Intra Uterine Insemination. ${ }^{18}$ The absolute number of motile sperms available for treatment purposes does not differ in the Swim up and Density gradient Group greatly. ${ }^{21}$
Swim up is very economical, ${ }^{16}$ easy to learn and perform technique which concentrates most of the motile spermatozoa present in the original neat semen sample but its efficiency in concentrating the normal morphological population is not significant. The Swim Up preparation in addition had some cellular debris in the yield. ${ }^{18}$ 
Density Gradient preparation on the other hand not only concentrated the motile spermatozoa with equal efficiency in addition it also enhanced the yield of available normal morphological sperms better. ${ }^{18}$ In addition we noticed the colloidal silane coated silica particles offered the particulate sieve mechanism and filtered effectively the cellular debris coming in to the yield. Thus the yield had few to nil cellular debris.

Running cost of the Swim Up and Density Gradient preparations when compared they did differ marginally in comparison to each other but differed heavily in comparison to the acquisition and running costs of other sperm processing methods ${ }^{16}$ In our study too we second this fact.

In one prospective comparative study published in 2016 the DGC method resulted in recovering up to $51 \%$ motile sperms against max $37 \%$ motile sperms in the yield, ${ }^{21}$ but, in our study we didn't see such trend. In a Cochrane Review ${ }^{22}$ published in 2014 to analyze the effect sperm parameters and success in IUI resulted in identifying lacunae in standardized testing methods, Huge heterogeneity in treatment modalities and an urgent and need for better cohort studies

\section{Conclusion}

From the above study it can be concluded that Discontinuous Density Gradient Sperm Preparation method should be the choice of sperm preparation as this method enhances available morphologically normal spermatozoa in yield better at a marginally high cost in comparison to Swim Up. Also the method yields cleaner fraction of spermatozoa with less debris.

In future such comparative study can be done on yield, in the diagnosis of sperm DNA Fragmentation, Oxidative Stress, longevity (Sperm survival assay) etc.,

Such Comparative study can be proposed to compare the outcome in an ART center with different treatment modalities like IUI/ IVF/ ICSI before accepting it as a standard method of sperm preparation in the facility.

\section{Acknowledgments and Declaration}

During the course of this study we obtained no monetary benefits or in kind benefits from the manufacturers/ dealers of the media and disposables. Institutional Ethical committee clearance was taken prior to undertaking the study.

\section{References}

1. Mahadevan M, Baker G. Assessment and preparation of semen for in vitro fertilization. In: Wood C, Trounson A. editor. Clinical In Vitro Fertilization. Springer-Verlag, Berlin; 1984. pp. 83-97.

2. World Health Organisation (2010) WHO laboratory manual for the Examination and processing of human semen 5 Edition Cambridge University Press, Cambridge.

3. Dicky RP, Pyrzak R, Lu PY, Taylor SN, Rye PH. Comparison of the sperm quality necessary for successful intrauterine insemination with world Health organization threshold values for normal sperm. Fertil Steril 1999;71:684-9.

4. Menkveld, R., Oettle, E.E., Kruger, T.F. et al. (1991) In Brown, C-L. (ed.), Atlas of Human Sperm Morphology. Williams \& Wilkins, Baltimore, p. 117.

5. World Health Organization (1992) WHO Laboratory Manual for the Examination of Human Semen and Sperm-Cervical Mucus Interaction, 3rd edition. Cambridge University Press, Cambridge.

6. World Health Organization (1999) WHO Laboratory Manual for Examination of Human Semen. Cambridge University Press, Cambridge.

7. Esmaeilzadeh S, Farsi M, Bijany A. Effects of sperm morphology on pregnancy rate in IUI cycles. J Reprod Infertil. 2007;8(3):205-12.

8. Isik Tasdemir, et. al., Effect of abnormal sperm head morphology on the outcome of intracytoplasmic sperm injection in humans.Human Reproduction vol.12 no.6 pp.1214-7,1997.

9. Sun, Y., Li, B., Fan, L. Q., Zhu, W. B., Chen, X. J., Feng, J. H., Yang, C. L. and Zhang, Y. H. (2012), Does sperm morphology affect the outcome of intrauterine insemination in patients with normal sperm concentration and motility?. Andrologia, 44: 299-304. doi:10.1111/j.1439-0272.2012.01280.

10. Nikbakht R, Saharkhiz N. The Influence of Sperm Morphology, Total Motile Sperm Count of Semen and the Number of Motile Sperm Inseminated in Sperm Samples on the Success of Intrauterine Insemination. International Journal of Fertility \& Sterility. 2011;5(3):168-73.

11. Henkel RR, Schill WB. Sperm preparation for ART. Reprod Biol Endocrinol 2003;1:108.

12. Babbo, C.J., Hecht, B.R. and Jjeyendan, R.S. (1999) Increased recovery of swim-up spermatozoa by application of 'antigravitational' centrifugation. Fertil. Steril., 72,556-8.

13. Bolton VN, Braude PR. Preparation of human spermatozoaatozoa for in vitro fertilization by isopycnic centrifugation on self generating density gradients. Arch Androl 1984;13:167-76.

14. Dirican EK, Ozgün OD, Akarsu S, Akın KO, Ercan O, Uğurlu M, Camsarı C, Kanyılmaz O, Kaya A, Unsal A. Clinical outcome of magnetic activated cell sorting of non-apoptotic spermatozoa before density gradient centrifugation for assisted reproduction. J Assist Reprod Genet 2008;25:375-81.

15. Said TM, Agarwal A, Grunewald S, Rasch M, Glander HJ, Paasch U. Evaluation of sperm recovery following annexin $\mathrm{V}$ magnetic-activated cell sorting separation. Reprod BioMed Online 2006;13:336-9.

16. Katz P, Nachtigall R, Showstack J. The economic impact of the assisted reproductive technologies. Nat Cell Biol 2002;4:29-32.

17. Menkveld, R., Stander, F.S., Kotze, T.J., Kruger, T.F. and van Zyl, J.A. (1990) The evaluation of morphological characteristics of human spermatozoa according to stricter criteria. Hum. Reprod., 5, 586-92.

18. Menkveld, R., Swanson, R.J., Kotze, T.J.vW. et al. (1990) Comparison of a discontinuous Percoll gradient method versus a swim-up method: effects on sperm morphology and other semen parameters. Andrologia, 22,152-8.

19. Inaudi P, Petrilli S, Joghtapour A, Trusso P and Petraglia F. Reduction of steps in the preparation of motile sperm for intrauterine insemination does not reduce efficacy of the procedure: simplified one-step swim-up method 
versus classic swim-up. Hum. Reprod. May 2002;17:1288-91.

20. McClure, R.D., Nunes, L. and Tom, R. (1989) Semen manipulation: improved sperm recovery and function with a two-layer Percoll gradient. Fertil. Steril., 51,874-7.

21. Butt A. Chohan MA (2016 Aug) Comparative Efficacy of desity gradient and swim-up methods of semen preparation in intrauterine insemination cycles. $J$ Pak Med Assoc: 66(8):932-7.

22. Ombelet W, Dhont N, Thijssen A et. al., Semen quality and prediction of IUI success in male subfertility: a systematic review. Reprod Biomed Online. 2014 Mar;28(3):300-9. 